\title{
Impact of community oriented interventions on pediatric care-seeking practices- A multi-country study in Cambodia, Guatemala, Kenya And Zambia
}

\author{
Anbrasi Edward $^{1 *}$, Juan F Sanchez ${ }^{2}$, Chea Chhorvann ${ }^{3}$, Christina Bowles ${ }^{4}$, Sydney Malama ${ }^{5}$ and Jane Chege ${ }^{6}$ \\ ${ }^{1}$ Department of International Health, Johns Hopkins University, USA \\ ${ }^{2}$ Doctoral Candidate, Johns Hopkins University, USA \\ ${ }^{3}$ Director, National Institute of Public Health, Cambodia \\ ${ }^{4}$ Senior Research Coordinator, Johns Hopkins University, USA \\ ${ }^{5}$ Lecturer, Institute of Economic and Social Research, Zambia \\ ${ }^{6}$ Senior Director, World Vision International, USA
}

\begin{abstract}
Appropriate pediatric care-seeking for common childhood illnesses in rural communities continues to be a major challenge in low and middle-income settings. A quasi-experimental study with matched comparison groups was designed to determine the effect of community oriented interventions on illness care-seeking for children in Cambodia, Kenya, Guatemala, and Zambia.

Study Design: Four districts or sub districts in each country were assigned to the intervention and comparison arms. Child caretakers in the intervention sites received timed counseling by community health workers (CHWs), and social accountability mechanisms including community scorecards were established to support community and health facility governance. Community councils were enhanced in all study sites, and comparison sites received routine CHW services.

Results: At the end of the study, facility care-seeking for fever and diarrhea (and ARI in Zambia), improved significantly in intervention sites for Cambodia and Zambia (Cambodia, Fever, 52.7\%-64.1\%, p<0.001, Diarrhea, 27.1\%-63.8\%, p<0.001, Zambia, ARI, 29\%-69.2\%, p<0.001, Fever, 30\%-79.3\%, p<0.001, Diarrhea, 29.1\%-77.4\%, p<0.001) but they were not greater than the improvements in the comparison sites (Cambodia, Fever, 59.9\%-75.5\%, p<0.001, Diarrhea, 39.6\%-79.1\%, p<0.001, Zambia, ARI, 6.3\%-70.3\%, p<0.001, Fever, 11.2\%-53\%, $\mathrm{p}<0.001$, Diarrhea, $4.6 \%-65.3 \%, \mathrm{p}<0.001)$. Caretakers in the wealthiest quintiles reported significantly higher odds of facility care-seeking in all countries, except Zambia (Cambodia 2.19 , 95\%CI:1.39-3.45, p<0.010, Guatemala, 3.97, 95\%CI:2.02-7.79, p<0.001, Kenya, 1.91, 95\%CI:1.01-3.64, p<0.050). Comorbidities, child age, and caretaker's education also emerged as predictors, but varied by country. The odds of facility care-seeking was higher in the comparison sites for Cambodia and Guatemala and intervention sites for Kenya and Zambia.

Conclusion: The findings illustrate the contextual variations in facility care-seeking, but effective community based strategies can enhance coverage. Continued efforts for strengthening community based systems are warranted to ensure community demand and equitable service utilization for diseases that contribute to major mortality burden for children in rural communities.
\end{abstract}

\begin{abstract}
Abbreviations: ARI: Acute Respiratory Illness; CHW: Community Health Worker; IMCI: Integrated Management of Childhood Illness; iCCM: Integrated Community Case Management; NGO: NonGovernmental Organization
\end{abstract}

\section{Introduction}

Despite the accelerated efforts to address the Millennium Development Goal of reducing mortality among children underfive, many countries failed to achieve their targets. An estimated 5.6 million deaths occurred in 2016, mainly due to preventable or treatable conditions, with pneumonia, malaria and diarrhea contributing to $40 \%$ of the disease burden in Sub Saharan Africa [1]. Evidence-based costeffective interventions have been developed to reduce child deaths, yet effective delivery of these interventions has been impeded by a myriad of household, systemic, and healthcare environment related factors. Inequities in access and optimal quality of curative care remains a challenge for the poorest and remotely located communities that have minimal access to health facilities [2].

The ability of child caretakers to recognize illness symptoms and seek appropriate care is essential in reducing morbidity and mortality burden especially in low and middle-income countries, and therefore prioritized by WHO's and UNICEF's Global Action Plan for the Control of Pneumonia and Diarrhea [3]. Where facility care is inaccessible, women seek care for children from the informal health sector including traditional healers, drug sellers, and community health workers (CHWs), especially in countries like Zambia [4,5]. A majority of the children do not receive appropriate and timely treatment within 24 hours of the onset of symptoms, which contributes to high levels of mortality [6-8].

WHO and UNICEF launched the Integrated Management of Childhood Illness (IMCI) and later instituted community case management (iCCM), to effectively address the major disease burden in children, but most health systems face formidable challenges in

Correspondence to: Anbrasi Edward, Department of International Health, Johns Hopkins University, USA, E-mail: aedward1@jhu.edu

Key words: pediatric care-seeking, community-oriented healthcare, community health workers, Cambodia, Guatemala, Kenya, Zambia

Received: February 02, 2018; Accepted: February 16, 2018; Published: February 21,2018 

Zambia

achieving coverage and effective delivery of care for these interventions. A key recommendation that emerged from an IMCI multi-country evaluation was for strong community based approaches to augment health service delivery systems, as well as greater accountability for coverage at the population level. There is a growing body of evidence that community based interventions engaging trained CHWs can effectively improve appropriate prevention and utilization of health services and careseekin behavior [10-12]. The recent momentum on enhancing the performance of CHWs to meet the demands for community based healthcare is also fraught with challenges, primarily due to the lack of effective support mechanisms to ensure their capacity and sustainability [13]. Recognition of illness symptoms by child caretakers and appropriate and timely illness care-seeking was prioritized as one of the three central tenets for IMCI, and also highlighted as a priority by the Global Action Plan for Control of Pneumonia and Diarrhea.

The need for reporting on effectiveness of large scale programs and prioritizing the commitment to evaluation has been cited to be of paramount importance, particularly where significant investments have been made [14,15]. Donor agencies are increasingly under pressure to illustrate accountability of resources expended through rigorous quantitative evaluations. For the past five decades, World Vision, a Christian Development, Relief and Advocacy organization, has made substantive investments in community based health and development interventions, focusing on populations living in extreme poverty. World Vision implements multifaceted development programs including the training and support of CHWs, and strengthening local leaders and community councils to empower communities and enhance health service utilization. This research was planned with the primary objective of determining the impact of a package of community oriented interventions on care-seeking behavior for ARI (Acute Respiratory Infection), diarrhea and fever that contribute to a high mortality burden in children under-five.

\section{Materials and methods}

The research study was conducted collaboratively by Johns Hopkins University, the National Institute of Public Health in Cambodia, Institute of Nutrition of Central America and Panama in Guatemala, Moi University School of Public Health in Kenya, and the Institute of Economic and Social Research at the University of Zambia.

\section{Study design}

The research was designed as a quasi-experimental non-randomized study in Cambodia, Guatemala, Kenya, and Zambia. In most contexts World Vision implements health and development programs through Area Development Programs (ADP) which include comprehensive interventions that integrate various packages of health, nutrition, water and sanitation, food security, education, and child sponsorship/ protection activities An ADP is a distinctive geographic area, representing a district or sub-district, with a population ranging from 19,000 to 25,000 people. In each study country, four ADPs were selected; two assigned to the intervention arm and two ADPs matched on select demographic and health characteristics to the comparison arm (Table 1). The intervention sites received a package of interventions including; a) household health promotion by CHWs through timed and targeted counseling based on stage of pregnancy and early childhood and b) establishment of social accountability mechanisms with Community Voice and Action and Community Scorecards, designed to strengthen community to health facility linkages, enhance community knowledge
Table 1. Selected study sites in each country -districts/sub districts.

\begin{tabular}{|l|c|c|c|c|}
\hline Study Arm & Cambodia & Guatemala & Kenya & Zambia \\
\hline Intervention & Chulkiri & Comapa & Karemo & Luampa \\
\hline Intervention & Pouk & Nuevo Amanecer & Katito & Magoye \\
\hline Comparison & Prasath Balang & Apas & Kegonga-Ntimaru & Choongo \\
\hline Comparison & Tbeng Meanchey & Tinamit Junam & Magunga & Nyimba \\
\hline
\end{tabular}

of health facility entitlements and support facility performance [16]. Facility and community management councils were established, or existing councils or committees were strengthened through training and supportive supervision based on the Global Fund's Community Systems Strengthening Framework [17], by World Vision in both the intervention and comparison sites. Communities in the comparison ADPs received routine services from the government, World Vision and other Non-Governmental Organizations (NGOs), including CHW services.

Baseline and final evaluations were conducted between 20132014 and 2016-2017, respectively. To eliminate bias due to seasonal variations, the evaluations were conducted in the same months at baseline and final for each country. Employing a multi-stage sampling strategy, communities were sampled in proportion to population size, and eligible households were selected from each sampling unit. In each household one eligible women between the ages of 15-49, and one child under-five were selected randomly. At baseline, up to three children under-five were included in each household. Required sample size was calculated to detect a significant difference in differences in stunting in children under-five, with a two-sided alpha of $.05(=0.05)$ and power of $80 \%(1-=0.80)$, with appropriate considerations for non-response rate $(5 \%)$ and design effect of 1.2. Illness episodes two weeks prior to the interview and subsequent care-seeking practices were elicited from child caretakers.

Standardized instruments used by the Demographic Health Surveys [18], were modified for the study. Structured interviews were administered to the head of households to obtain data on sociodemographic characteristics, food security, etc. Subsequently, women who delivered in the previous two years and child caretakers were interviewed to obtain information on reproductive history, and care-seeking practices for maternal, newborn and child health. Survey teams, were trained on survey field procedures, informed consent protocols, and research ethics. Teams conducted the assessments with close supervision. Standard protocols for translation, field testing, survey procedures including participant confidentiality were followed during data collection.

The study was reviewed and approved by the Johns Hopkins Bloomberg School of Public Health's Institutional Review Board and the respective ethical and research review boards of the research institutions in each country.

\section{Data analysis}

Data from the baseline and final evaluations was cleaned, verified and analyzed using STATA v14.2 [19]. We first performed a descriptive analysis computing averages across the intervention and comparison sites for data from the final evaluation. To determine significance between interventions we performed $t$ tests between intervention and comparison and baseline and final results. These were not controlled for secular trends. A difference in difference analysis was computed using the procedure described by Gertler et al. [20]. Univariate and multivariate logistic regression models were used to determine predictors of care-seeking for data from the final evaluation. Cases 
with missing data for the independent variables were excluded from the regression models. Wealth quintiles were constructed employing a principle component analysis with a combination of 12 household assets (television, radio, bicycle, etc.) and household type (roofing, drinking water source, type of sanitation, etc.). Collinearity was tested for the independent variables used in the regression models.

\section{Results}

Results from the final evaluation are described here, with a baseline and final difference in difference analysis to determine effectiveness of the intervention.

\section{Characteristics of study population}

Table 2 summarizes characteristics of the study population for the final evaluation in the four countries. Majority of the households ( $>85 \%)$ were headed by males, except in Zambia, where a high proportion of households were headed by females, especially in the intervention sites (55.5\%). Significant differences were also evident in mean family size in all countries between intervention and comparison sites, which ranged between 4.7 and 5.6. In most of these rural households, the caretakers of the children under-five were female, the majority between the ages of 20-36 years, and over $25 \%$ of the female caretakers were either single, divorced or widowed in Zambia and Guatemala. Female caretakers in Guatemala had the lowest educational levels, with more than $25 \%$ reporting no formal education.

\section{Illness care-seeking patterns by study site}

Table 3 illustrates the illness care-seeking patterns of children for ARI, diarrhea and fever for the four countries following the intervention. Caretaker description of symptoms was used to define diarrhea and fever. ARI was defined as caretaker report of symptoms of cough with difficult or rapid breathing. Most caretakers (>90\%) sought care for ARI, fever and diarrhea for Cambodia, in both intervention and comparison sites, but $17.1 \%$ reported not seeking any care for diarrhea in the comparison sites. About $20 \%$ of the caretakers in Cambodia reported seeking care from drug vendors or pharmacies in the intervention sites. None of the caretakers reported seeking care from $\mathrm{CHWs}$ or health posts in the interventions sites, and $<5 \%$ reported seeking care from these providers in the comparison sites. For Guatemala, about $40 \%$ reported not seeking care for ARI, fever, and diarrhea for both intervention and comparison sites, and likewise health post care-seeking ranged from $21.8 \%$ to $32.2 \%$. CHW careseeking was low $(<3 \%)$. Few caretakers in Kenya reported symptoms of ARI (26 in intervention and 22 in comparison sites); of these, a higher proportion reported not seeking care in the comparison sites (15.4\% vs. $31.8 \%)$. Health posts were mentioned by three caretakers, and four reported pharmacies and drug sellers in the intervention sites. Significant differences were evident for seeking care for fever between intervention and comparison sites ( $11.1 \%$ vs. $42.9 \%)$. Health posts, CHW house and pharmacy were more frequently mentioned by caretakers in the intervention than comparison sites as locations of care for fever. Likewise, for diarrhea, $36.3 \%$ vs. $46 \%$ did not seek care for intervention and comparison sites, and health post, and pharmacy was mentioned more frequently in the intervention sites. A higher proportion of caretakers from the intervention sites (18\%-27.7\%) in Zambia, reported not seeking care for ARI, fever and diarrhea than the comparison sites (9.3\%-18.2\%). However, a higher proportion of the caretakers reported seeking care from health posts, in the comparison sites compared to the intervention sites.

Table 4 presents the reported illness two weeks preceding the survey and facility care-seeking of children under-five for the four countries for both baseline and final data with a time trend and difference in

Table 2. Sociodemographic characteristics of study population-final evaluation. I: Intervention; C: Comparison.

\begin{tabular}{|c|c|c|c|c|c|c|c|c|c|c|c|c|}
\hline \multirow{3}{*}{ Characteristics } & \multicolumn{3}{|l|}{$\begin{array}{l}\text { Cambodia } \\
\mathrm{N}=2954\end{array}$} & \multicolumn{3}{|l|}{$\begin{array}{l}\text { Guatemala } \\
\mathbf{N}=\mathbf{3 1 4 0}\end{array}$} & \multicolumn{3}{|l|}{\begin{tabular}{|l} 
Kenya \\
N=3515
\end{tabular}} & \multicolumn{3}{|l|}{$\begin{array}{l}\text { Zambia } \\
\mathrm{N}=\mathbf{2 1 4 9}\end{array}$} \\
\hline & I & $\mathrm{C}$ & & I & $\mathrm{C}$ & & I & $\mathrm{C}$ & & I & $\mathrm{C}$ & \\
\hline & $\mathrm{N}(\%)$ & $\mathrm{N}(\%)$ & p-value & $\mathrm{N}(\%)$ & $\mathrm{N}(\%)$ & p-value & $\mathrm{N}(\%)$ & $\mathrm{N}(\%)$ & p-value & $\mathrm{N}(\%)$ & $\mathrm{N}(\%)$ & p-value \\
\hline Male-headed households & $1283(87.8)$ & $1329(89.7)$ & 0.100 & $1382(89.4)$ & $1427(89.5)$ & 0.905 & $1534(85.2)$ & $1477(87.9)$ & 0.022 & $462(44.5)$ & $759(70.2)$ & 0.000 \\
\hline Mean family size & 5.1 & 4.8 & 0.000 & 4.8 & 5.1 & 0.000 & 4.9 & 4.7 & 0.000 & 5.6 & 5.1 & 0.000 \\
\hline \multicolumn{13}{|l|}{ Child caretaker gender } \\
\hline Male & $3(0.2)$ & - & 0.083 & $20(1.3)$ & $16(1.0)$ & 0.442 & $88(4.9)$ & $111(6.9)$ & 0.013 & $51(5.1)$ & $64(6.1)$ & 0.334 \\
\hline Female & $1458(99.8)$ & $1487(100.0)$ & 0.083 & $1521(98.7)$ & $1577(99.0)$ & 0.442 & $1718(95.1)$ & $1501(93.1)$ & 0.013 & $952(94.9)$ & $992(93.9)$ & 0.334 \\
\hline \multicolumn{13}{|l|}{ Female caretaker age } \\
\hline $15-19 y$ & $50(3.4)$ & $71(4.8)$ & 0.066 & $97(6.4)$ & $129(8.2)$ & 0.053 & $158(9.2)$ & $124(8.3)$ & 0.348 & $106(11.1)$ & $110(11.1)$ & 0.974 \\
\hline $20-36 y$ & $1164(79.8)$ & $1288(86.6)$ & 0.000 & $1125(74.0)$ & $1157(73.4)$ & 0.706 & $1374(80.0)$ & $1252(83.4)$ & 0.012 & $661(69.4)$ & $724(73.0)$ & 0.084 \\
\hline $37-49 y$ & $156(10.7)$ & $121(8.1)$ & 0.017 & $254(16.7)$ & $251(15.9)$ & 0.555 & $119(6.9)$ & $74(4.9)$ & 0.016 & $150(15.8)$ & $133(13.4)$ & 0.143 \\
\hline \multicolumn{13}{|l|}{ Female caretaker marital status } \\
\hline Married & $1320(96.6)$ & $1437(97.3)$ & 0.261 & $963(65.3)$ & $874(56.9)$ & 0.000 & $1386(87.2)$ & $1338(92.8)$ & 0.000 & $598(70.0)$ & $708(74.8)$ & 0.023 \\
\hline Single/Divorced/Widowed & $47(3.4)$ & $40(2.7)$ & 0.261 & $511(34.7)$ & $661(43.1)$ & 0.000 & $203(12.8)$ & $103(7.2)$ & 0.000 & $254(29.7)$ & $236(24.9)$ & 0.023 \\
\hline \multicolumn{13}{|l|}{ Female caretaker education } \\
\hline No education & $239(17.4)$ & $357(24.1)$ & 0.000 & $461(31.2)$ & $429(27.9)$ & 0.046 & $16(1.0)$ & $84(5.8)$ & 0.000 & $73(8.0)$ & $157(16.2)$ & 0.000 \\
\hline Primary & $728(53.1)$ & $652(44.0)$ & 0.000 & $799(54.1)$ & 904 (58.8) & 0.010 & $1171(70.9)$ & $1055(72.9)$ & 0.232 & $456(49.8)$ & 479 (49.6) & 0.914 \\
\hline Secondary or more & $394(28.8)$ & $466(31.5)$ & 0.113 & $213(14.4)$ & $202(13.1)$ & 0.305 & $456(27.6)$ & $290(20.0)$ & 0.000 & $384(42.0)$ & $320(33.1)$ & 0.000 \\
\hline Biological mother of child & $1332(91.0)$ & $1472(99.0)$ & 0.000 & $1446(93.6)$ & $1511(94.8)$ & 0.151 & $1784(98.5)$ & $1649(97.5)$ & 0.028 & $979(95.9)$ & $1031(96.4)$ & 0.507 \\
\hline \multicolumn{13}{|l|}{ Child age } \\
\hline $0-11 \mathrm{~m}$ & $482(32.9)$ & $566(38.0)$ & 0.004 & $368(23.8)$ & $340(21.3)$ & 0.098 & $665(36.5)$ & $469(27.7)$ & 0.000 & $287(27.3)$ & $246(22.4)$ & 0.008 \\
\hline $12-23 \mathrm{~m}$ & $544(37.1)$ & $613(41.2)$ & 0.025 & $331(21.4)$ & $343(21.5)$ & 0.941 & $659(36.2)$ & 541 (31.9) & 0.007 & $274(26.1)$ & $231(21.0)$ & 0.006 \\
\hline $24-59 \mathrm{~m}$ & $439(30.0)$ & $310(20.8)$ & 0.000 & 847 (54.8) & 911 (57.2) & 0.182 & $496(27.3)$ & 685 (40.4) & 0.000 & 489 (46.6) & $622(56.6)$ & 0.000 \\
\hline
\end{tabular}

I Intervention; C Comparison 
Edward A (2018) Impact of community oriented interventions on pediatric care-seeking practices- A multi-country study in Cambodia, Guatemala, Kenya And Zambia

Table 3. Source of care for Acute Respiratory Infection (ARI), fever and diarrhea-final evaluation. p-value not computed for low samples.

\begin{tabular}{|c|c|c|c|c|c|c|c|c|c|c|c|c|}
\hline \multirow{3}{*}{ Source of Care } & \multicolumn{3}{|l|}{$\begin{array}{l}\text { Cambodia } \\
\mathrm{N}=\mathbf{2 9 5 4}\end{array}$} & \multicolumn{3}{|c|}{$\begin{array}{l}\text { Guatemala } \\
\mathbf{N}=\mathbf{3 1 4 0}\end{array}$} & \multicolumn{3}{|l|}{$\begin{array}{l}\text { Kenya } \\
N=3515\end{array}$} & \multicolumn{3}{|l|}{$\begin{array}{l}\text { Zambia } \\
\text { N=2149 }\end{array}$} \\
\hline & \multirow{2}{*}{\begin{tabular}{|l|}
$\mathrm{I}$ \\
$\mathrm{N}(\%)$ \\
\end{tabular}} & \multicolumn{2}{|l|}{ C } & \multirow{2}{*}{$\begin{array}{l}\mathrm{I} \\
\mathrm{N}(\%)\end{array}$} & \multicolumn{2}{|l|}{$\mathrm{C}$} & \multirow{2}{*}{$\begin{array}{l}\mathrm{I} \\
\mathrm{N}(\%)\end{array}$} & \multicolumn{2}{|l|}{$\mathrm{C}$} & \multirow{2}{*}{\begin{tabular}{|l|}
$\mathrm{I}$ \\
$\mathrm{N}(\%)$
\end{tabular}} & \multicolumn{2}{|l|}{$\mathrm{C}$} \\
\hline & & $\mathrm{N}(\%)$ & $\mathrm{p}$-value & & $\mathrm{N}(\%)$ & p-value & & $\mathrm{N}(\%)$ & p-value & & $\mathrm{N}(\%)$ & p-value \\
\hline Reported ARI in past 2 weeks & 102 & 85 & & 160 & 142 & & 26 & 22 & & 50 & 43 & \\
\hline Did not seek care & $2(2.0)$ & $6(7.1)$ & - & $69(43.1)$ & $68(47.9)$ & 0.409 & $4(15.4)$ & $7(31.8)$ & - & $9(18.0)$ & $4(9.3)$ & - \\
\hline Government clinic & $25(24.5)$ & $15(17.6)$ & 0.252 & $6(3.8)$ & $5(3.5)$ & - & $10(38.5)$ & $14(63.6)$ & - & $26(52.0)$ & $3(7.0)$ & - \\
\hline Government hospital & $16(15.7)$ & $10(11.8)$ & - & $8(5.0)$ & $16(11.3)$ & - & $2(7.7)$ & - & - & $0(0.0)$ & $2(4.6)$ & - \\
\hline Private hospital/clinic & $22(21.6)$ & $38(44.7)$ & 0.001 & $14(8.7)$ & 15 (10.6) & - & $2(7.7)$ & - & - & $2(4.0)$ & $22(51.2)$ & - \\
\hline Health post & - & - & - & $50(31.3)$ & $31(21.8)$ & 0.064 & $3(11.5)$ & - & - & $7(14.0)$ & $7(16.3)$ & - \\
\hline CHW & - & $1(1.1)$ & - & - & - & - & - & - & - & $1(2.0)$ & $3(7.0)$ & - \\
\hline Pharmacy & $24(23.5)$ & $9(10.6)$ & 0.017 & $13(8.1)$ & $4(2.8)$ & - & $4(15.4)$ & $1(4.6)$ & - & $2(4.0)$ & $1(2.3)$ & - \\
\hline $\begin{array}{l}\text { Other (home remedy, traditional medicine, } \\
\text { nurse home etc.) }\end{array}$ & $13(12.7)$ & $6(7.1)$ & - & - & $3(2.1)$ & - & - & - & - & $2(4.0)$ & - & - \\
\hline Reported fever in past 2 weeks & 507 & 565 & & 233 & 266 & & 180 & 112 & & 173 & 209 & \\
\hline Did not seek care & $19(3.7)$ & $49(8.7)$ & 0.001 & $93(39.9)$ & $108(40.6)$ & 0.876 & $20(11.1)$ & $48(42.9)$ & 0.000 & $48(27.7)$ & $38(18.2)$ & 0.028 \\
\hline Government clinic & $161(31.7)$ & $110(19.4)$ & 0.000 & $10(4.3)$ & $2(0.8)$ & - & $57(31.7)$ & $38(33.9)$ & 0.691 & $88(50.9)$ & $29(13.9)$ & 0.000 \\
\hline Government hospital & $51(10.1)$ & $32(5.7)$ & 0.008 & $6(2.6)$ & $18(6.8)$ & - & $23(12.8)$ & $2(1.8)$ & - & $3(1.7)$ & $10(4.8)$ & - \\
\hline Private hospital/clinic & $93(18.3)$ & $244(43.2)$ & 0.000 & $26(11.2)$ & $28(10.5)$ & 0.821 & $11(6.1)$ & $5(4.4)$ & - & $4(2.3)$ & $51(24.4)$ & 0.000 \\
\hline Health post & - & $11(1.9)$ & - & $73(31.3)$ & $83(31.2)$ & 0.976 & $16(8.9)$ & $1(0.9)$ & - & $16(9.2)$ & $54(25.8)$ & 0.000 \\
\hline CHW & - & $23(4.1)$ & - & - & $3(1.1)$ & - & $5(2.8)$ & $1(0.9)$ & - & $2(1.2)$ & $18(8.6)$ & - \\
\hline Pharmacy & $115(22.7)$ & $58(10.3)$ & 0.000 & $24(10.3)$ & $15(5.6)$ & 0.058 & $29(16.1)$ & $5(4.4)$ & 0.001 & $4(2.3)$ & $2(0.9)$ & - \\
\hline $\begin{array}{l}\text { Other (home remedy, mother, neighbor, } \\
\text { traditional medicine, provider's home) }\end{array}$ & $67(13.2)$ & $38(6.7)$ & 0.000 & $1(0.4)$ & $9(3.4)$ & - & $13(7.2)$ & $9(8.0)$ & - & $2(1.2)$ & $3(1.4)$ & - \\
\hline Reported diarrhea in past 2 weeks & 145 & 187 & & 124 & 137 & & 160 & 100 & & 135 & 183 & \\
\hline Did not seek care & $12(8.3)$ & $32(17.1)$ & 0.014 & $64(51.6)$ & $69(50.4)$ & 0.841 & $58(36.3)$ & $46(46.0)$ & 0.123 & $33(24.4)$ & $32(17.5)$ & 0.137 \\
\hline Government clinic & $39(26.9)$ & $32(17.1)$ & 0.035 & $5(4.0)$ & $4(2.9)$ & - & $47(29.4)$ & $32(32.0)$ & 0.658 & $65(48.1)$ & $40(21.9)$ & 0.000 \\
\hline Government hospital & $11(7.6)$ & $14(7.5)$ & - & $1(0.8)$ & $8(5.8)$ & - & $14(8.8)$ & $2(2.0)$ & - & $2(1.5)$ & $6(3.3)$ & - \\
\hline Private hospital/clinic & $35(24.1)$ & $76(40.6)$ & 0.001 & $7(5.7)$ & $9(6.6)$ & - & $11(6.9)$ & $8(8.0)$ & - & $7(5.2)$ & $37(20.2)$ & 0.000 \\
\hline Health post & - & $3(1.6)$ & - & $40(32.2)$ & $37(27.0)$ & 0.356 & $6(3.8)$ & $1(1.0)$ & - & $11(8.1)$ & $30(16.4)$ & 0.023 \\
\hline CHW & - & $5(2.7)$ & - & - & $4(2.9)$ & - & $4(2.5)$ & $3(3.0)$ & - & $5(3.7)$ & $12(6.6)$ & - \\
\hline Pharmacy & $31(21.4)$ & $14(7.5)$ & 0.000 & $7(5.7)$ & $5(3.7)$ & - & $14(8.8)$ & $4(4.0)$ & - & $5(3.7)$ & $1(0.5)$ & - \\
\hline Other & $16(11.0)$ & $11(5.9)$ & - & - & $1(0.7)$ & - & $3(1.9)$ & $1(1.0)$ & - & - & - & - \\
\hline
\end{tabular}

p-value not computed for low samples

difference analysis. A significantly lower proportion of caretakers reported symptoms of fever and diarrhea in both intervention and comparison sites following the intervention for Cambodia and Guatemala. Report of ARI symptoms was also significantly lower for Cambodia. The pattern was similar in Kenya and Zambia, except that levels of reported diarrhea were slightly higher for the intervention sites in Kenya and comparison sites in Zambia at final evaluation. The difference in difference analysis from baseline and final evaluations however indicated that this was only significant for diarrhea between intervention and comparison sites in Cambodia, ARI for Guatemala, and fever and diarrhea for Kenya and Zambia. The proportion reporting comorbidities was significantly lower at the end of the study, in all countries except Kenya, where the values were lower, but not significant.

Facility care-seeking was defined as those who sought care from private or public clinics or hospitals, but not health posts. Though iCCM is practiced in some contexts where CHWs provide first level treatment for diarrhea, ARI and fever, this was not standardized in the study sites. Facility care-seeking for fever and diarrhea was significantly higher in both intervention and comparison sites at the end of the study than at baseline for Cambodia. Care-seeking practices also showed similar patterns in Guatemala, though significance levels were only evident for comparison sites. Likewise, comparison sites showed better improvements in Kenya, with declining levels for ARI in Intervention sites. The improvements in care-seeking practices were highest for Zambia from 30\% at baseline to about $70 \%$ for ARI, fever and diarrhea for children in the intervention sites, though the comparison sites also showed remarkable improvements for most illness symptoms. Facility care-seeking was about $60-70 \%$ for the three main illnesses for Cambodia, Kenya, and Zambia, with Guatemala reporting the lowest levels $(21.7 \%-48.6 \%)$ at the end of the study. The increases in proportions of care-seeking were almost similar in intervention and comparison sites overall, resulting in statistically insignificant difference in differences. Except for the comparison sites in Kenya, prompt care-seeking (within one day), was proportionately higher for all countries, in the intervention and comparison sites

\section{Treatment for ARI, fever and diarrhea}

Antibiotic treatment for children with ARI symptoms (cough and difficult/rapid breathing) for suspected pneumonia, as reported by caretakers (Table 5), was high for Cambodia in both sites, though significantly higher in intervention sites ( $99 \%$ vs. $78.2 \%, \mathrm{p}<0.001)$, less than $50 \%$ for Guatemala, and even lower for Kenya $(<40 \%)$, and significantly higher for comparison sites in Zambia (54\% vs. 79.1 , $\mathrm{p}=0.010$ ). A significantly higher proportion of caretakers reported treatment of fever with antimalarials for Guatemala, Kenya, and 
Edward A (2018) Impact of community oriented interventions on pediatric care-seeking practices- A multi-country study in Cambodia, Guatemala, Kenya And Zambia

Table 4. Illness care-seeking patterns in intervention and comparison sites before and after the study in Cambodia, Kenya, Guatemala and Zambia. ARI: Acute Respiratory Infection; B Baseline; F: Final; DD: Difference in difference.

\begin{tabular}{|c|c|c|c|c|c|c|c|c|c|c|c|c|c|c|c|c|}
\hline \multirow{3}{*}{ Illness Care-seeking } & \multicolumn{4}{|c|}{ Cambodia } & \multicolumn{4}{|c|}{ Guatemala } & \multicolumn{4}{|c|}{ Kenya } & \multicolumn{4}{|c|}{ Zambia } \\
\hline & \multirow{2}{*}{$\begin{array}{l}\text { B } \\
\%\end{array}$} & \multirow{2}{*}{\begin{tabular}{|l|}
$\mathrm{F}$ \\
$\%$
\end{tabular}} & \multicolumn{2}{|c|}{$\mathrm{p}$ value } & \multirow{2}{*}{\begin{tabular}{|l|} 
B \\
$\%$
\end{tabular}} & \multirow{2}{*}{\begin{tabular}{|l|}
$\mathrm{F}$ \\
$\%$
\end{tabular}} & \multicolumn{2}{|l|}{$\mathrm{p}$ value } & \multirow{2}{*}{\begin{tabular}{|l|} 
B \\
$\%$
\end{tabular}} & \multirow{2}{*}{\begin{tabular}{|l}
$\mathrm{F}$ \\
$\%$
\end{tabular}} & \multicolumn{2}{|c|}{$\mathrm{p}$ value } & \multirow{2}{*}{$\begin{array}{l}\text { B } \\
\%\end{array}$} & \multirow{2}{*}{$\begin{array}{l}\mathrm{F} \\
\%\end{array}$} & \multicolumn{2}{|c|}{$\mathrm{p}$ value } \\
\hline & & & $\begin{array}{l}\text { Time } \\
\text { trend }\end{array}$ & DD & & & $\begin{array}{l}\text { Time } \\
\text { trend }\end{array}$ & $\mathrm{DD}$ & & & $\begin{array}{l}\text { Time } \\
\text { trend }\end{array}$ & DD & & & $\begin{array}{l}\text { Time } \\
\text { trend }\end{array}$ & DD \\
\hline \multicolumn{17}{|c|}{$\begin{array}{l}\text { Reported illness in past } 2 \\
\text { weeks }\end{array}$} \\
\hline \multicolumn{17}{|c|}{$\begin{array}{l}\text { ARI (cough with difficult } \\
\text { breathing) }\end{array}$} \\
\hline Intervention & 10.4 & 7.0 & 0.001 & \multirow[t]{2}{*}{0.548} & 12.6 & 10.3 & 0.052 & 0.006 & 3.0 & 1.4 & 0.001 & 0.838 & 14.9 & 4.7 & 0.000 & 0.716 \\
\hline Comparison & 10.1 & 5.9 & 0.000 & & 15.5 & 8.9 & 0.000 & & 2.7 & 1.3 & 0.002 & & 13.4 & 4.0 & 0.000 & \\
\hline Fever & & & & & & & & & & & & & & & & \\
\hline Intervention & 44.6 & 34.5 & 0.000 & 0.860 & 20.4 & 15.1 & 0.000 & 0.494 & 19.5 & 9.9 & 0.000 & 0.000 & 32.3 & 16.6 & 0.000 & 0.000 \\
\hline Comparison & 48.1 & 38.5 & 0.000 & & 23.4 & 16.7 & 0.000 & & 10.9 & 6.6 & 0.000 & & 10.8 & 19.4 & 0.000 & \\
\hline Diarrhea & & & & & & & & & & & & & & & & \\
\hline Intervention & 18.0 & 9.8 & 0.000 & 0.001 & 17.8 & 8.0 & 0.000 & 0.760 & 8.3 & 8.8 & 0.518 & 0.012 & 17.7 & 12.9 & 0.001 & 0.000 \\
\hline Comparison & 27.0 & 12.6 & 0.000 & & 17.8 & 8.6 & 0.000 & & 8.2 & 5.9 & 0.004 & & 14.5 & 16.8 & 0.108 & \\
\hline Diarrhea and blood in & & & & & & & & & & & & & & & & \\
\hline Intervention & 8.0 & 6.9 & 0.679 & 0.429 & 11.6 & 12.1 & 0.880 & 0.550 & 10.9 & 8.2 & 0.369 & 0.979 & 18.6 & 12.2 & 0.105 & 0.000 \\
\hline Comparison & 9.9 & 5.9 & 0.100 & & 9.2 & 12.5 & 0.282 & & 16.9 & 14.1 & 0.523 & & 8.7 & 27.9 & 0.000 & \\
\hline $\begin{array}{l}\text { Comorbidities ( } \geq 2 \text { illn } \\
\text { reported) }\end{array}$ & & & & & & & & & & & & & & & & \\
\hline Intervention & 20.4 & 14.3 & 0.000 & 0.013 & 13.5 & 9.1 & 0.000 & 0.002 & 5.6 & 4.0 & 0.010 & 0.256 & 30.5 & 5.6 & 0.000 & 0.000 \\
\hline Comparison & 24.1 & 13.3 & 0.000 & & 16.3 & 7.0 & 0.000 & & 3.4 & 2.8 & 0.207 & & 13.2 & 7.4 & 0.001 & \\
\hline Sought facility care & & & & & & & & & & & & & & & & \\
\hline ARI & & & & & & & & & & & & & & & & \\
\hline Intervention & 81.5 & 63.0 & 0.006 & 0.030 & 23.7 & 30.8 & 0.278 & 0.001 & 78.2 & 66.7 & 0.273 & 0.010 & 29.0 & 69.2 & 0.000 & 0.058 \\
\hline Comparison & 79.5 & 81.0 & 0.812 & & 12.6 & 48.6 & 0.000 & & 60.9 & 93.3 & 0.018 & & 6.3 & 70.3 & 0.000 & \\
\hline Fever & & & & & & & & & & & & & & & & \\
\hline Intervention & 52.7 & 64.1 & 0.001 & 0.350 & 22.3 & 30.0 & 0.116 & 0.094 & 68.1 & 64.1 & 0.357 & 0.000 & 30.0 & 79.3 & 0.000 & 0.300 \\
\hline Comparison & 59.9 & 75.5 & 0.000 & & 12.4 & 30.4 & 0.000 & & 58.7 & 88.2 & 0.000 & & 11.2 & 53.0 & 0.000 & \\
\hline Diarrhea & & & & & & & & & & & & & & & & \\
\hline Intervention & 27.1 & 63.8 & 0.000 & 0.684 & 19.4 & 21.7 & 0.716 & 0.049 & 79.6 & 72.4 & 0.169 & 0.068 & 29.1 & 77.4 & 0.000 & 0.078 \\
\hline Comparison & 39.6 & 79.1 & 0.000 & & 12.6 & 30.9 & 0.001 & & 75.5 & 84.0 & 0.209 & & 4.6 & 65.3 & 0.000 & \\
\hline $\begin{array}{l}\text { Sought Immediate car } \\
\text { (within } 1 \text { day) }\end{array}$ & & & & & & & & & & & & & & & & \\
\hline ARI & & & & & & & & & & & & & & & & \\
\hline Intervention & 16.9 & 48.0 & 0.000 & 0.013 & 4.6 & 20.0 & 0.002 & 0.178 & 16.2 & 30.0 & 0.169 & 0.834 & 17.7 & 25.6 & 0.341 & 0.002 \\
\hline Comparison & 40.9 & 45.6 & 0.544 & & 1.1 & 24.3 & 0.000 & & 10.8 & 21.4 & 0.325 & & 4.2 & 48.6 & 0.000 & \\
\hline Fever & & & & & & & & & & & & & & & & \\
\hline Intervention & 18.6 & 49.7 & 0.000 & 0.000 & 5.2 & 13.6 & 0.009 & 0.914 & 17.9 & 31.8 & 0.000 & 0.011 & 17.3 & 36.5 & 0.000 & 0.606 \\
\hline Comparison & 36.6 & 48.5 & 0.000 & & 0.9 & 8.9 & 0.000 & & 20.9 & 15.6 & 0.408 & & 9.5 & 25.3 & 0.001 & \\
\hline Diarrhea & & & & & & & & & & & & & & & & \\
\hline Intervention & 14.3 & 51.5 & 0.000 & 0.150 & 4.5 & 6.7 & 0.540 & 0.917 & 20.0 & 38.8 & 0.001 & 0.039 & 19.9 & 46.7 & 0.000 & 0.883 \\
\hline Comparison & 25.6 & 53.6 & 0.000 & & 2.8 & 4.5 & 0.507 & & 17.3 & 18.0 & 0.915 & & 3.3 & 29.0 & 0.000 & \\
\hline Total Sample & B & & $\mathrm{F}$ & & B & & $\mathrm{F}$ & & B & & $\mathrm{F}$ & & B & & $\mathrm{F}$ & \\
\hline Intervention & 3202 & & 1466 & & 4013 & & 1546 & & 3689 & & 1820 & & 3754 & & 1062 & \\
\hline Comparison & 3494 & & 1480 & & 4144 & & 1594 & & 3268 & & 1695 & & 3182 & & 1100 & \\
\hline
\end{tabular}

ARI Acute Respiratory Infection B Baseline F Final DD Difference in difference

Zambia in intervention than comparison sites (Guatemala, 13.3\% vs. $7.1 \%$, p $=0.025$, Kenya $63.9 \%$ vs. $38.4 \%, p=0.001$, Zambia $24.8 \%$ vs. $11.5 \%, \mathrm{p}=0.001)$. Antimalarial treatment was not reported by caretakers in Cambodia. Only three caretakers reported seeking care from traditional healers in Kenya, and therefore this category was not included in the table figures.

Continued feeding during diarrhea was reported by more than $60 \%$ of the caretakers in Cambodia, and more than $30 \%$ in Zambia, with significantly higher proportions reporting continuing feeding in the intervention sites for Guatemala and Kenya (Guatemala, 25\% vs. $12.4 \%$, $\mathrm{p}<0.010$, Kenya, $40 \%$ vs. $21 \%$, $\mathrm{p}<0.001)$. Caretakers in the intervention sites in Zambia, reported significantly higher levels of administering more fluids for children during diarrhea, than in the comparison sites ( $40 \%$ vs. $25.1 \%$, p $<0.010$ ). Likewise, for Kenya and Cambodia, higher levels were reported in intervention sites, but not significant compared to the comparison sites. For Guatemala, significantly higher levels were reported in comparison sites ( $30.6 \%$ vs. $46.7 \%$, p < 0.010$)$. For both Kenya and Zambia, oral rehydration therapy (ORT) treatment for diarrhea was above $70 \%$, with intervention sites reporting higher proportions, but not significantly different from comparison sites. Caretakers from comparison sites in Guatemala also reported high levels of ORT use (73.7\%), slightly lower (67.8\%) for intervention sites. The figures were 
Edward A (2018) Impact of community oriented interventions on pediatric care-seeking practices- A multi-country study in Cambodia, Guatemala, Kenya And Zambia

Table 5. Reported treatment for Acute Respiratory Infection (ARI), fever and diarrhea- final evaluation. ORT: Oral Rehydration Therapy; I: Intervention; C: Comparison; p-value not computed for low samples.

\begin{tabular}{|c|c|c|c|c|c|c|c|c|c|c|c|c|}
\hline \multirow{3}{*}{ Characteristics } & \multicolumn{3}{|l|}{$\begin{array}{l}\text { Cambodia } \\
\mathrm{N}=2946\end{array}$} & \multicolumn{3}{|l|}{$\begin{array}{l}\text { Guatemala } \\
\mathbf{N}=\mathbf{3 1 4 0}\end{array}$} & \multicolumn{3}{|l|}{\begin{tabular}{|l|} 
Kenya \\
$\mathrm{N}=3515$
\end{tabular}} & \multicolumn{3}{|l|}{\begin{tabular}{|l} 
Zambia \\
$\mathrm{N}=\mathbf{2 1 4 9}$
\end{tabular}} \\
\hline & \multirow{2}{*}{\begin{tabular}{|l}
$\mathrm{I}$ \\
$\mathrm{N}(\%)$
\end{tabular}} & \multicolumn{2}{|l|}{$\mathrm{C}$} & \multirow{2}{*}{$\begin{array}{l}\text { I } \\
\mathrm{N}(\%)\end{array}$} & \multicolumn{2}{|l|}{$\mathrm{C}$} & \multirow{2}{*}{\begin{tabular}{|l}
$\mathrm{I}$ \\
$\mathrm{N}(\%)$
\end{tabular}} & \multicolumn{2}{|l|}{$\mathrm{C}$} & \multirow{2}{*}{\begin{tabular}{|l}
$\mathrm{I}$ \\
$\mathrm{N}(\%)$
\end{tabular}} & \multicolumn{2}{|l|}{$\mathrm{C}$} \\
\hline & & $\mathrm{N}(\%)$ & $\mathrm{p}$-value & & $\mathrm{N}(\%)$ & p-value & & $\mathrm{N}(\%)$ & p-value & & $\mathrm{N}(\%)$ & $\mathrm{p}$-value \\
\hline Reported ARI & $102(7.0)$ & $87(5.9)$ & 0.232 & $160(10.3)$ & $142(8.9)$ & 0.173 & $26(1.4)$ & $22(1.3)$ & 0.735 & $50(4.7)$ & $43(4.0)$ & 0.400 \\
\hline \multicolumn{13}{|l|}{ Treatment for ARI } \\
\hline Antibiotics & $101(99.0)$ & $68(78.2)$ & 0.000 & $74(46.3)$ & $70(49.3)$ & 0.598 & $8(30.8)$ & $8(36.4)$ & - & $27(54.0)$ & $34(79.1)$ & 0.010 \\
\hline Reported Fever & $506(34.5)$ & $567(38.5)$ & 0.026 & $233(15.1)$ & $266(16.7)$ & 0.213 & $180(9.9)$ & $112(6.6)$ & 0.000 & $173(16.6)$ & 209 (19.4) & 0.085 \\
\hline \multicolumn{13}{|l|}{ Treatment for fever } \\
\hline Antimalarials & - & - & - & $31(13.3)$ & $19(7.1)$ & 0.025 & $115(63.9)$ & $43(38.4)$ & 0.000 & $43(24.8)$ & $24(11.5)$ & 0.001 \\
\hline Reported diarrhea & $144(9.8)$ & $186(12.6)$ & 0.018 & $124(8.0)$ & $137(8.6)$ & 0.553 & $160(8.8)$ & $100(5.9)$ & 0.001 & $135(12.9)$ & $183(16.8)$ & 0.012 \\
\hline \multicolumn{13}{|l|}{ Treatment for diarrhea } \\
\hline Continued feeding & $99(68.8)$ & $118(63.4)$ & 0.313 & $31(25.0)$ & $17(12.4)$ & 0.010 & $64(40.0)$ & $21(21.0)$ & 0.001 & $52(38.5)$ & $61(33.3)$ & 0.344 \\
\hline More fluids & $73(50.7)$ & 77 (41.4) & 0.094 & $38(30.6)$ & $64(46.7)$ & 0.008 & $34(21.3)$ & $13(13.0)$ & 0.080 & $54(40.0)$ & $46(25.1)$ & 0.006 \\
\hline ORT (any form) & $84(58.3)$ & $120(64.5)$ & 0.255 & $84(67.7)$ & $101(73.7)$ & 0.291 & $117(73.1)$ & $68(68.0)$ & 0.383 & $109(80.7)$ & $134(73.2)$ & 0.113 \\
\hline Zinc & $5(3.5)$ & $6(3.2)$ & - & $1(0.8)$ & $4(2.9)$ & - & $27(16.9)$ & $11(11.0)$ & 0.176 & $1(0.7)$ & $21(11.5)$ & - \\
\hline Antibiotic & $80(55.6)$ & $43(23.1)$ & 0.000 & $16(12.9)$ & $14(10.2)$ & 0.501 & $13(8.1)$ & $5(5.0)$ & - & $17(12.6)$ & $42(22.9)$ & 0.015 \\
\hline Antimotility & $0(0.0)$ & $2(1.1)$ & - & $8(6.4)$ & $37(27.0)$ & 0.000 & $0(0.0)$ & $1(1.0)$ & - & $0(0.0)$ & $8(4.4)$ & - \\
\hline Intravenous fluids & $10(6.9)$ & $10(5.4)$ & - & $0(0.0)$ & $0(0.0)$ & - & $0(0.0)$ & $0(0.0)$ & - & $1(0.7)$ & $0(0.0)$ & - \\
\hline Home remedy & $3(2.1)$ & $12(6.4)$ & - & $12(9.7)$ & $14(10.2)$ & - & $8(5.0)$ & $6(6.0)$ & - & $5(3.7)$ & $3(1.6)$ & - \\
\hline
\end{tabular}

ORT Oral Rehydration Therapy, I Intervention, C Comparison

p-value not computed for low samples

lower for Cambodia $(<65 \%)$. Except for Kenya, and comparison sites for Zambia, zinc treatment for diarrhea was $<5 \%$ for all other study sites. More than half the caretakers reported antibiotic treatment for diarrhea in intervention sites in Cambodia. Antibiotic treatment was proportionately lower for Guatemala and Kenya $(<13 \%)$. Antimotility drugs, intravenous fluids, and home remedies were also mentioned by a small proportion of caretakers $(<10 \%)$, except for antimotility drugs in the comparison sites for Guatemala (27\%).

\section{Predictors of illness care-seeking}

We first performed a univariate analysis to determine significance of associations for each predictor variable selected. Those that were significant were then included for the multivariate logistic regression

(Table 6). Records with key missing data $(<10 \%)$ were excluded from the regression analysis. As the healthcare context is varied, analysis was performed by country. iCCM has been introduced in some of these sites, but there was no available documentation of its implementation by CHWs. Hence, we included two models, facility care-seeking and facility and $\mathrm{CHW}$ or health post care-seeking. Caretaker marital status and age, significant in the univariate analysis, were not significant predictors in the multivariate model. Comorbidities emerged as a significant predictor of facility care-seeking for all countries for both models except for Zambia for intervention sites, where the odds were higher but not significant. Likewise, caretakers from highest wealth quintile had higher odds of seeking facility care than caretakers from poorer quintiles in all countries (Cambodia 2.19, 95\% CI:1.39 -3.45, $\mathrm{p}<0.010$, Guatemala 3.97, 95\% CI:2.02-7.79, $\mathrm{p}<0.001$, Kenya 1.91, 95\%CI:1.01-3.64, p<0.050, Zambia 1.41, 95\%CI:0.75-2.63, NS). The odds were also significantly higher for care-seeking when CHW and health posts were included in the care-seeking framework, for caretakers in higher quintiles for Cambodia, but not for other countries. Caretakers with younger children $(<24 \mathrm{~m})$ had a higher odds of facility care-seeking for illness, than those with older children for Cambodia and Guatemala. Though the odds of facility care-seeking were higher for caretakers with primary or secondary school education compared to those with no education in Guatemala and Kenya, it was statistically significant only for those with secondary school education in Guatemala. Adjusting for all known confounders, intervention sites had higher odds of care-seeking for Kenya and Zambia though not significant. Intervention sites showed lower odds of facility careseeking, for Cambodia and Guatemala (Cambodia, 0.58, 95\%CI:0.44 0.75), $\mathrm{p}<0.001$, Guatemala, 0.62, 95\%CI:0.41-0.93, $\mathrm{p}<0.010)$.

Table 7 presents the regression outcomes by wealth quintile and type of illness reported by country. Caretakers from wealthier quintiles had significantly higher odds for facility care-seeking for ARI and fever for Cambodia and Guatemala than the poorer quintiles adjusting for marital status, educational levels, caretaker age, child age, comorbidities, and intervention groups. Facility care-seeking showed higher odds based on wealth quintiles for Kenya and Zambia, but not to the level of significance.

\section{Discussion}

A myriad of healthcare environment and household level factors influence illness care-seeking practices in rural communities. Findings from this study provide some evidence of improved facility-based care for all countries in both intervention and comparison sites except for ARI in Cambodia and Kenya.

An earlier systematic review on care-seeking behaviors illustrated varied patterns of illness incidence between Asia and Africa. ARI and fever symptoms were more frequently reported in Asia [21]. This study showed a higher proportion of care-seeking for ARI and fever than diarrhea for Cambodia, although proportions reporting no care were almost similar for fever and diarrhea for Kenya and Zambia. This may be related to perceived illness severity for ARI and fever than diarrhea, by the child caretakers. As observed in the systematic review, care from private clinics or hospitals were reported by more than $40 \%$ of the caretakers in the comparison sites in Cambodia, than the intervention sites $(<25 \%)$. This may be partly attributed to the CHW health promotion and community engagement strategies including Community Voice and Action and Community Scorecards in the intervention sites, encouraging service utilization and measures 
Edward A (2018) Impact of community oriented interventions on pediatric care-seeking practices- A multi-country study in Cambodia, Guatemala, Kenya And Zambia

Table 6. Odds of care-seeking for Acute Respiratory Infection (ARI), fever and diarrhea at public or private facilities and CHW/health post by country-final evaluation. *p $<0.05, * * \mathrm{p}<0.01$, $* * * \mathrm{p}<0.001$ Facility (Public or private clinic, facility or hospital) CHW (Community Health Worker) HP (Health Post).

\begin{tabular}{|c|c|c|c|c|c|c|c|c|}
\hline & \multirow{2}{*}{\multicolumn{2}{|c|}{\begin{tabular}{|l|} 
Cambodia \\
$\mathrm{OR}(95 \% \mathrm{CI})$ \\
\end{tabular}}} & \multicolumn{2}{|l|}{ Guatemala } & \multicolumn{2}{|l|}{ Kenya } & \multicolumn{2}{|l|}{ Zambia } \\
\hline & & & \multirow{2}{*}{$\begin{array}{l}\text { OR }(95 \% \mathrm{CI}) \\
\text { Facility }\end{array}$} & \multirow{2}{*}{\begin{tabular}{|l|} 
OR $(95 \% \mathrm{CI})$ \\
Facility/CHW/HP \\
\end{tabular}} & \multirow{2}{*}{$\begin{array}{l}\text { OR }(95 \% \mathrm{CI}) \\
\text { Facility }\end{array}$} & \multirow{2}{*}{\begin{tabular}{|l} 
OR(95\% CI $)$ \\
Facility/CHW/HP
\end{tabular}} & \multirow{2}{*}{$\begin{array}{l}\text { OR }(95 \% \mathrm{CI}) \\
\text { Facility }\end{array}$} & \multirow{2}{*}{\begin{tabular}{|l}
$\mathrm{OR}(95 \% \mathrm{CI})$ \\
Facility/CHW/HP \\
\end{tabular}} \\
\hline & Facility & Facility/CHW/HP & & & & & & \\
\hline $\begin{array}{l}\text { Marital status (ref, single/divorced/ } \\
\text { widowed) }\end{array}$ & 1.00 & 1.00 & 1.00 & 1.00 & 1.00 & 1.00 & 1.00 & 1.00 \\
\hline Married & $\begin{array}{l}0.99(0.52- \\
1.88)\end{array}$ & $1.03(0.53-1.98)$ & $\begin{array}{l}1.36(0.88- \\
2.09)\end{array}$ & $0.99(0.73-1.36)$ & $\begin{array}{l}0.89(0.48- \\
1.65)\end{array}$ & $1.12(0.60-2.08)$ & $\begin{array}{l}0.83(0.55- \\
1.25)\end{array}$ & $0.70(0.43-1.14)$ \\
\hline Caretaker education (ref, none) & 1.00 & 1.00 & 1.00 & 1.00 & 1.00 & 1.00 & 1.00 & 1.00 \\
\hline Primary & $\begin{array}{l}0.72(0.51- \\
1.03)\end{array}$ & $0.62(0.42-0.90) *$ & $\begin{array}{l}1.25(0.77- \\
2.03)\end{array}$ & $1.14(0.81-1.60)$ & $\begin{array}{l}1.58(0.44- \\
5.67)\end{array}$ & $2.11(0.59-7.58)$ & $\begin{array}{l}0.97(0.57- \\
1.64)\end{array}$ & $0.79(0.42-1.50)$ \\
\hline Secondary or more & $\begin{array}{l}0.95(0.63- \\
1.43)\end{array}$ & $0.76(0.50-1.18)$ & $\begin{array}{l}1.97(1.03- \\
3.76)^{*}\end{array}$ & $1.22(0.73-2.02)$ & $\begin{array}{l}1.43(0.37- \\
5.49)\end{array}$ & $1.84(0.48-7.05)$ & $\begin{array}{l}1.00(0.56- \\
1.83)\end{array}$ & $0.84(0.42-1.71)$ \\
\hline Caretaker age (ref, <24y) & 1.00 & 1.00 & 1.00 & 1.00 & 1.00 & 1.00 & 1.00 & 1.00 \\
\hline$\geq 24 y$ & $\begin{array}{l}1.06(0.79- \\
1.44)\end{array}$ & $0.98(0.72-1.34)$ & $\begin{array}{l}1.33(0.82- \\
2.17)\end{array}$ & $1.31(0.92-1.87)$ & $\begin{array}{l}0.86(0.56- \\
1.31)\end{array}$ & $1.01(0.65-1.55)$ & $\begin{array}{l}0.76(0.51- \\
1.11)\end{array}$ & $1.02(0.66-1.58)$ \\
\hline Child age (ref, <24m) & 1.00 & 1.00 & 1.00 & 1.00 & 1.00 & 1.00 & 1.00 & 1.00 \\
\hline$\geq 24 \mathrm{~m}$ & $\begin{array}{l}0.62(0.45- \\
0.85)^{* *}\end{array}$ & $\begin{array}{l}0.62(0.45- \\
0.85)^{* *}\end{array}$ & $\begin{array}{l}0.64(0.43- \\
0.95)^{*}\end{array}$ & $0.70(0.52-0.94)^{*}$ & $\begin{array}{l}0.97(0.62- \\
1.54)\end{array}$ & $1.02(0.65-1.62)$ & $\begin{array}{l}1.14(0.80- \\
1.62)\end{array}$ & $0.83(0.55-1.24)$ \\
\hline Comorbidities (ref, one symptom) & 1.00 & 1.00 & 1.00 & 1.00 & 1.00 & 1.00 & 1.00 & 1.00 \\
\hline 2 or more symptoms & $\begin{array}{l}1.78(1.35- \\
2.34)^{* * *}\end{array}$ & $\begin{array}{l}1.75(1.32- \\
2.32)^{* * *}\end{array}$ & $\begin{array}{l}1.54(1.02- \\
2.33)^{*}\end{array}$ & $1.53(1.11-2.09)^{* *}$ & $\begin{array}{l}2.00(1.24- \\
3.22)^{* *}\end{array}$ & $\begin{array}{l}2.14(1.31- \\
3.50)^{* *}\end{array}$ & $\begin{array}{l}1.52(0.98- \\
2.35)\end{array}$ & $\begin{array}{l}2.62(1.46- \\
4.69)^{* *}\end{array}$ \\
\hline Wealth quintile (ref, lowest) & 1.00 & 1.00 & 1.00 & 1.00 & 1.00 & 1.00 & 1.00 & 1.00 \\
\hline Low & $\begin{array}{l}1.24(0.86- \\
1.78)\end{array}$ & $1.16(0.80-1.69)$ & $\begin{array}{l}0.95(0.44- \\
2.02)\end{array}$ & $0.87(0.54-1.41)$ & $\begin{array}{l}0.99(0.53- \\
1.89)\end{array}$ & $1.11(0.59-2.10)$ & $\begin{array}{l}0.81(0.48- \\
1.36)\end{array}$ & $0.65(0.35-1.20)$ \\
\hline Middle & $\begin{array}{l}1.27(0.88- \\
1.85)\end{array}$ & $1.27(0.86-1.86)$ & $\begin{array}{l}1.52(0.77- \\
3.02)\end{array}$ & $0.66(0.42-1.05)$ & $\begin{array}{l}1.04(0.53- \\
2.02)\end{array}$ & $1.07(0.55-2.09)$ & $\begin{array}{l}0.96(0.55- \\
1.70)\end{array}$ & $0.77(0.40-1.48)$ \\
\hline High & $\begin{array}{l}1.60(1.07- \\
2.38)^{* *}\end{array}$ & $1.54(1.02-2.33)^{*}$ & $\begin{array}{l}1.71(0.86- \\
3.42)\end{array}$ & $0.79(0.49-1.27)$ & $\begin{array}{l}1.25(0.68- \\
2.32)\end{array}$ & $1.14(0.61-2.11)$ & $\begin{array}{l}1.00(0.59- \\
1.71)\end{array}$ & $0.67(0.36-1.24)$ \\
\hline Highest & $\begin{array}{l}2.19(1.39- \\
3.45)^{* *}\end{array}$ & $\begin{array}{l}2.08(1.31- \\
3.31)^{* *}\end{array}$ & $\begin{array}{l}3.97(2.02- \\
7.79)^{* * *}\end{array}$ & $0.93(0.56-1.53)$ & $\begin{array}{l}1.91(1.01- \\
3.64)^{*}\end{array}$ & $1.63(0.85-3.13)$ & $\begin{array}{l}1.41(0.75- \\
2.63)\end{array}$ & $0.87(0.42-1.79)$ \\
\hline Treatment (ref, Comparison) & 1.00 & 1.00 & 1.00 & 1.00 & 1.00 & 1.00 & 1.00 & 1.00 \\
\hline Intervention & $\begin{array}{l}0.58(0.44- \\
0.75)^{* * *}\end{array}$ & $\begin{array}{l}0.45(0.34- \\
0.58)^{* * *}\end{array}$ & $\begin{array}{l}0.62(0.41- \\
0.93)^{*}\end{array}$ & $0.88(0.65-1.19)$ & $\begin{array}{l}1.11(0.72- \\
1.71)\end{array}$ & $1.46(0.95-2.27)$ & $\begin{array}{l}1.33(0.93- \\
1.90)\end{array}$ & $\begin{array}{l}0.57(0.38- \\
0.86)^{* *}\end{array}$ \\
\hline Constant & $\begin{array}{l}1.85(0.86- \\
3.98)\end{array}$ & $\begin{array}{l}2.97(1.35- \\
6.53)^{* *}\end{array}$ & $\begin{array}{l}0.09(0.04- \\
0.20)^{* * *}\end{array}$ & $0.96(0.56-1.65)$ & $\begin{array}{l}0.51(0.12- \\
2.18)\end{array}$ & $0.31(0.07-1.36)$ & $\begin{array}{l}1.12(0.54- \\
2.32)\end{array}$ & $\begin{array}{l}6.42(2.65- \\
15.5)^{* * *}\end{array}$ \\
\hline $\mathrm{N}$ & 1107 & 1107 & 747 & 747 & 389 & 389 & 536 & 536 \\
\hline
\end{tabular}

$* \mathrm{p}<0.05, * * \mathrm{p}<0.01, * * * \mathrm{p}<0.001$ Facility (Public or private clinic, facility or hospital) CHW (Community Health Worker) HP (Health Post)

Table 7. Odds of facility care-seeking (public or private hospitals, clinics or facilities) by illness and by household wealth quintile-final evaluation. $*$ p $<0.05$, $* * \mathrm{p}<0.01, * * * \mathrm{p}<0.001$. Model adjusted for marital status, caretaker education, caretaker age, child age, comorbidities, and intervention group.

\begin{tabular}{|c|c|c|c|c|}
\hline Care-seeking by illness & $\begin{array}{l}\text { Cambodia } \\
\text { OR }(95 \% \text { CI) }\end{array}$ & $\begin{array}{l}\text { Guatemala } \\
\text { OR }(95 \% \text { CI })\end{array}$ & $\begin{array}{l}\text { Kenya } \\
\text { OR }(95 \% \text { CI) }\end{array}$ & \begin{tabular}{|l|} 
Zambia \\
OR $(95 \%$ CI $)$
\end{tabular} \\
\hline ARI (ref, lowest) & $1.00(\mathrm{~N}=180)$ & $1.00(\mathrm{~N}=292)$ & $1.00(\mathrm{~N}=38)$ & $1.00(\mathrm{~N}=81)$ \\
\hline Low & $2.05(0.82-5.14)$ & $0.51(0.13-1.96)$ & $0.45(0.05-4.29)$ & $1.49(0.31-7.11)$ \\
\hline Middle & $2.56(0.97-6.78)$ & $1.16(0.40-3.35)$ & $0.47(0.39-5.73)$ & $4.71(0.67-33.24)$ \\
\hline High & $5.17(1.54-17.30)^{* *}$ & $3.28(1.18-9.09)^{*}$ & $1.16(0.13-10.52)$ & $1.03(0.26-3.99)$ \\
\hline Highest & $2.24(0.57-8.87)$ & $4.77(1.69-13.43)^{* *}$ & $0.75(0.09-6.21)$ & $2.46(0.44-13.81)$ \\
\hline Fever (ref, lowest) & $1.00(\mathrm{~N}=1032)$ & $1.00(\mathrm{~N}=485)$ & $1.00(\mathrm{~N}=237)$ & $1.00(\mathrm{~N}=317)$ \\
\hline Low & $1.19(0.82-1.73)$ & $1.30(0.53-3.17)$ & $0.73(0.32-1.68)$ & $0.88(0.44-1.78)$ \\
\hline Middle & $1.30(0.88-1.92)$ & $1.75(0.76-4.06)$ & $0.54(0.22-1.35)$ & $0.60(0.27-1.33)$ \\
\hline High & $1.53(1.02-2.30)^{*}$ & $1.90(0.82-4.42)$ & $0.86(0.40-1.84)$ & $0.79(0.37-1.69)$ \\
\hline Highest & $1.96(1.23-3.11)^{* *}$ & $4.19(1.83-9.59)^{* *}$ & $1.25(0.55-2.78)$ & $1.52(0.64-3.60)$ \\
\hline Diarrhea (ref, lowest) & $1.00(\mathrm{~N}=319)$ & $1.00(\mathrm{~N}=256)$ & $1.00(\mathrm{~N}=225)$ & $1.00(\mathrm{~N}=259)$ \\
\hline Low & $1.47(0.75-2.90)$ & $0.90(0.21-3.92)$ & $1.43(0.60-3.40)$ & $1.09(0.51-2.32)$ \\
\hline Middle & $1.72(0.86-3.43)$ & $3.15(0.90-10.96)$ & $1.99(0.82-4.85)$ & $2.00(0.87-4.55)$ \\
\hline High & $1.54(0.69-3.41)$ & $1.18(0.28-4.95)$ & $2.37(0.99-5.66)$ & $1.33(0.64-2.77)$ \\
\hline Highest & $1.73(0.79-3.79)$ & $3.47(0.90-13.40)$ & $2.32(0.95-5.67)$ & $1.18(0.46-3.01)$ \\
\hline
\end{tabular}

$* \mathrm{p}<0.05, * * \mathrm{p}<0.01, * * * \mathrm{p}<0.001$

Model adjusted for marital status, caretaker education, caretaker age, child age, comorbidities, and intervention group 
to enhance provider trust and facility operations and performance, though causal associations cannot be made. Convenience, promptness of care, courteous staff, and perceived quality of care, have been previously reported as reasons for seeking care from private rather than public facilities [21].

The Ministry of Health in Zambia, in collaboration with the Malaria Consortium, has made enormous investments to train and equip CHWs to provide first level treatment especially for malaria, for children presenting with fever symptoms [22-24]. Unlike other studies in Zambia, where mothers preferred accessing care from CHWs, following the community case management intervention [5], this study showed that about $70 \%$ sought facility care for ARI, diarrhea, and fever. Most national health systems integrate CHWs in the health service delivery architecture, and include iCCM in their policies to screen and treat uncomplicated malaria, diarrhea, and ARI. But feedback from the communities indicated that the primary focus of CHWs was for preventive and referral measures rather than fist level curative care, except for treatment of diarrhea with ORT. Results from this study showed that only one caretaker in Cambodia, $20-30 \%$ caretakers in Guatemala, $11.5 \%$ in intervention sites in Kenya, and $2-16 \%$ for Zambia accessed care from CHWs or health posts for symptoms of ARI. Similar trends were evident for symptoms of fever and diarrhea for all countries as reported in other studies [21-25].

Consultations with traditional healers were rarely mentioned except for three cases in Kenya, but $20 \%$ of caretakers in the intervention sites reported accessing treatment from pharmacies and drug vendors for Cambodia, and $15 \%$ in intervention sites for fever and ARI for Kenya. Less than $5 \%$ of the caretakers mentioned drug vendors or pharmacies in Zambia. High rates of care-seeking from drug vendors has been reported for malaria especially in Africa [21], and other studies have effectively leveraged this behavior to enhance the skills of private drug retailers to prescribe artemisinin-based combination therapy for children presenting with these symptoms [26]. Another study in rural Kenya reported similar levels of treatment from traditional healers and community volunteers [27]. However, antibiotic treatment for ARI $(<40 \%)$, and diarrhea $(<10 \%)$, was much lower in this study, than that reported in the earlier study in Kenya $(60 \%)$. A few caretakers also reported receiving treatment in the homes of nurses or midwives and providing home remedies for their children. Despite the accelerated global advocacy efforts to integrate zinc in the treatment protocol for diarrhea, $<5 \%$ of the caretakers mentioned zinc, except for the intervention sites for Kenya (17\%), and comparison site in Zambia (12\%).

Caretaker recognition of illness symptoms and early care-seeking with appropriate skilled providers has been advocated as an essential prerequisite for preventing child mortality. Our study showed that in both intervention and comparison sites prompt care-seeking (within a day of onset of symptoms) for ARI, diarrhea, and cough improved in all countries, though levels were still below $50 \%$ for most. The efforts invested for $\mathrm{CHW}$ training and household health promotion, and community engagement strategies for enhancing service utilization and community and facility governance were effective in improving facility care-seeking and to a smaller extent early care-seeking in these rural households for both intervention and comparison sites. Additional efforts are needed to sustain these gains and to increase the levels of service utilization, as the three conditions of ARI, diarrhea, and malaria contribute to the major disease burden in these contexts.

Gender of head of household was not shown to be a significant factor of care-seeking in another study [28]. We found no association between male or female headed households in care-seeking for the bivariate analysis. Socioeconomic status has been shown to be positively correlated with care-seeking in other African countries [25,27,29-31]. Wealth quintile was a significant predictor of careseeking in this study for Cambodia and Guatemala, and though the odds were higher for the wealthier quintiles in other countries, it was not statistically significant. However, a similar health education strategy in Zambia showed that long distances and low household income no longer influenced careseeking practices following the behavior change intervention [32]. A study in China demonstrated that mothers with higher education had higher odds of care-seeking than those withlower educational status [33]. Similar patterns were observed in our study, for Guatemala, Kenya, and Zambia, but statistically significant only for women with secondary education for Guatemala. Gender of the child was not addressed in this study, but a systematic review on care-seeking behaviors reported mixed results on gender differentials in careseeking [21]. The systematic review also found differential careseeking practices for women from higher socioeconomic backgrounds who mostly consulted with private providers. Illness severity was not obtained in this study, but comorbidities were documented and illustrated significantly higher odds of care-seeking for most countries. Perceived illness severity was a determining factor for other studies for illness care-seeking [28,34]. In Kenya, studies reported higher facility care for younger children $(<11 \mathrm{~m})$, than older children [35].

It is apparent that the improvements in care-seeking between intervention and comparison sites were not very different for the four study countries. Several factors may have contributed to these results; a review of program implementation documents indicated that training interventions for CHWs were delayed in some of the intervention sites, reducing the expected duration of intervention. In Guatemala, long distances and worsening security was cited as one of the main impediments for CHWs to conduct household visits. Assessments of CHW systems in the intervention sites indicated poor support and supervision following training, and varying task expectations related to number of assigned households to CHWs. The comparison sites also supported CHWs to conduct household visits and provided counseling to mothers and caretakers, though the timing of the visits was not sequenced with appropriate messaging for maternal and child health, as it was in the intervention sites. Community and facility councils were closely engaged in health promotion activities in the comparison sites. The results from Zambia and Kenya showed higher odds of facility care in intervention sites, but were not significant. This may also be due to increased prompt referrals by CHWs in Kenya and Zambia, where iCCM is strongly advocated. Another large scale multicountry evaluation also reported no differences in child mortality for sites receiving an accelerated child survival intervention, attributing the lack of improvement to rapid changes in the contextual factors, lack of health systems capacity, and ineffective CHW systems, among others [36]. Another study in Bangladesh reported 20\% appropriate care-seeking in IMCI study sites and the institution of CHWs only demonstrated a $4 \%$ improvement, to a final coverage of $24 \%$ in the IMCI sites, though significantly higher than comparison sites [37].

A limitation of this research was the lack of information on the barriers to care-seeking, which was obtained through qualitative assessments from community stakeholders, but not included in the household surveys. These included geographical access, lack of transport, cost of services, objection from family members, etc.; these factors were also observed in other studies [21]. Another important barrier to care-seeking from public facilities may be attributed to poor quality of care, as children presenting in ambulatory clinics do 

Zambia

not receive optimal screening, diagnosis and treatment, even for the common IMCI conditions in these contexts [38]. Illness incidence was based on caretaker reports, which introduces another limitation, and illness severity was not recorded. The national guidelines for service delivery differ for each country, the metrics used in this study were not contextualized for these standards. CHWs in Cambodia, are not permitted to provide first level curative care, as mandated by iCCM, but offer support and advice on health-related issues, aside from preventive and health promotion activities.

Another important study limitation is the lack of a true control, as facilities and communities in the comparison sites were exposed to several systems strengthening mechanisms, including investments to enhance the functionality of facility and community councils by World Vision in all countries, a maternal and new born initiative in Zambia, which bolstered the skills and competencies of healthcare providers, trained $\mathrm{CHWs}$ and ensured system readiness and capacity for service delivery, and other developmental activities by NGOs operating in these sites. The health management committees in both intervention and comparison sites in Cambodia played a key role in improving healthcare service utilization, based on documentation provided in the program reports. Cambodia has also bolstered its efforts to enhance the quality of healthcare services since 2015, stimulating demand and service utilization. Though the training for the Community Voice and Action was distinctive from the training for community councils, often there was ambiguity about their roles and functions, and community councils performed similar functions for enhancing health facility operations, thus minimizing the real effect of the interventions. The healthcare context has been considered a key determinant as it can confound the effect of the interventions [36,39]. The characteristics of the health system, healthcare provider capacity and competencies, availability of essential commodities, policies, etc., were not factored in the analysis, which poses an additional limitation in the study inference. Worsening security in Comapa, an intervention site in Guatemala, and doctor's and nurse's strike in Kenya were reported during the study, and posed risks for CHWs and limited access to facility care.

Illness care-seeking and determinants varied between countries, but illustrated similar trends in terms of lower levels of care-seeking of poorer caretakers, higher levels for those children presenting with comorbidities, and caretaker education, and child age emerging as significant predictors for some countries. Community oriented programs must prioritize awareness and access for those from poorer economic backgrounds and with lower levels of education to ensure equitable care.

\section{Conclusion}

In conclusion, effective multidimensional community based strategies addressing household behaviors through effective $\mathrm{CHW}$ services, complemented by social accountability mechanisms for community and facility engagement can enhance service utilization, by promoting facility care-seeking and mitigating the barriers to access care. Except for the higher levels reported for some care-seeking practices, overall, we found that both intervention and comparison sites showed improvements in care-seeking as investments for strengthening the capacity of the district healthcare system and other ongoing efforts by World Vision and other NGOs, including the existing CHW systems, were as successful in improving health seeking behaviors in the comparison sites as the timed and targeted counseling from CHWs, and Community Voice and Action, in the intervention sites. Engaging private pharmacy and drug sellers and traditional healers in the community engagement strategies would ensure a more concerted effort for addressing child health in these communities in the future, especially in contexts where the practice is more evident, and within the policy structure. District health teams and NGOs investing in community based healthcare, must aim to create resilient service delivery systems engaging local stakeholders to determine feasible solutions to address the current barriers in care-seeking practices.

The study demonstrates that future programming for community based efforts must strengthen and effectively leverage community entities to target key communications, to address the complex dynamics of care-seeking practices, and increase demand for quality facility care. Efforts to improve the documentation of specific activities carried out by community councils, Community Voice and Action, CHWs, etc., and empirical research on their effectiveness will enable local government and development agencies to craft context specific programming and enhance equitable access to healthcare.

\section{Authorship and Contributions}

AE, JC, CC, and SM designed the study and CC and SM implemented the study. JFS performed the data analysis under the supervision of AE. $\mathrm{AE}$ wrote the manuscript with subsequent contributions and approval of CB, CC, JFS, JC, and SM for the final version.

\section{Acknowledgement}

We thank the research and survey teams from the National Institute of Nutrition, Cambodia, Institute of Economic and Social Research, Zambia, Institute of Nutrition of Central America and Panama, Guatemala and Moi University, Kenya. In particular, we would like to acknowledge the leadership and contributions of Manuel Ramirez-Zea, Kevin Martinez, Mubiana Macwan'gi, and Grace Ettyang, for the conduct of the research study, the district health staff, World Vision research and program staff, particularly Annette E Ghee and Bridget Adam, and Henry B Perry (Johns Hopkins University) for their continued support through the study. We also acknowledge with thanks, all the community members who participated in the study.

\section{Funding Information}

Funding for the study was from a grant from World Vision to Johns Hopkins University, with subcontracts to the National Institute of Nutrition, Cambodia, Institute of Economic and Social Research,

Zambia, Institute of Nutrition of Central America and Panama, Guatemala, and Moi University, Kenya.

\section{Competing Interests}

$\mathrm{AE}, \mathrm{CB}, \mathrm{CC}$, JFS, and SM, were partially funded by the grant. JC was employed by World Vision.

\section{References}

1. UN IGME (2017) Levels \& trends in child mortality: report 2017. Estimates developed by the UN Inter-agency Group for Child Mortality Estimation. New York: United Nations Children's Fund.

2. Schellenberg JA, Victora CG, Mushi A, De Savigny D, Schellenberg D, et al. (2003) Inequities among the very poor: health care for children in rural southern Tanzania. Lancet 361: 561-566. [Crossref]

3. UNICEF (2003) Communities and families-Improving knowledge of caregivers website.

4. Yeboah-Antwi K, Pilingana P, Macleod WB, Semrau K, Siazeele K, et al. (2010) Community case management of fever due to malaria and pneumonia in children under five in Zambia: a cluster randomized controlled trial. PLoS Med 7: 1000340. [Crossref] 
5. Seidenberg PD, Hamer DH, Iyer H, Pilingana P, Siazeele K, et al. (2012) Impact of integrated community case management on health-seeking behavior in rural Zambia. Am J Trop Med Hyg 87: 105-110. [Crossref]

6. UNICEF (2004) Management of pneumonia in community settings: WHO/UNICEF joint statement.

7. UNICEF, WHO (2006) Pneumonia: the forgotten killer of children. A Book. New York: The United Nations Children's Fund: 4-40.

8. Schellenberg, J, Victora C, Mushi A, De Savigny D, Schellenberg D, et al. (2003) Tanzania Integrated Management of Childhood Illness MCE Baseline Household Survey Study Group Inequities among the very poor: health care for children in rural southern Tanzania. Lancet 361: 561-566

9. Bryce J, Victora CG, Habicht JP, Black RE, Scherpbier RW (2005) Programmatic pathways to child survival: results of a multi-country evaluation of Integrated Management of Childhood Illness. Health Policy Plan 20: i5-i17. [Crossref]

10. Lassi ZS, Haider BA, Bhutta ZA (2010) Community-based intervention packages for reducing maternal and neonatal morbidity and mortality and improving neonatal outcomes. Cochrane Database Syst Rev 10: 11. [Crossref]

11. Bhutta ZA, Lassi ZS, Pariyo GW, Huicho L. Global Experience of Community Health Workers for Delivery of Health-Related Millennium Development Goals: A Systematic Review, Country Case Studies, and Recommendations for Integration into National Health Systems. In: WHO and Global Health Workforce Alliance, editor. Geneva; 2010

12. Bhutta ZA, Das JK, Walker N, Rizvi A, Campbell H, et al. (2013) Interventions to address deaths from childhood pneumonia and diarrhoea equitably: what works and at what cost? The Lancet 381: 1417-1429. [Crossref]

13. Naimoli JF, Perry HB, Townsend JW, Frymus DE, Mccaffery JA (2015) Strategic partnering to improve community health worker programming and performance: features of a community-health system integrated approach. Human resources for health 13:46.

14. Bryce J, Victora CG (2005) Child survival: countdown to 2015. The Lancet 365: 2153 2154

15. Horton R, Murray C, Frenk J (2008) A new initiative and invitation for health monitoring, tracking, and evaluation. Lancet 371: 1139-1140. [Crossref]

16. Schaaf M, Topp SM, Ngulube M (2017) From favours to entitlements: community voice and action and health service quality in Zambia. Health Policy Plan 32: 847-859. [Crossref]

17. Global Fund (2010) Community systems strengthening framework. Geneva: The Global Fund.

18. https://www.dhsprogram.com/What-We-Do/Survey-Types/DHS.cfm

19. (2015) Stata, version 15 [computer program]. Texas, USA: college station.

20. Gertler PJ, Martinez S, Premand P, Rawlings LB, Vermeersch CM (2016) Impact evaluation in practice. World Bank Publications. Washington, USA.

21. Geldsetzer P, Williams TC, Kirolos A, Mitchell S, Ratcliffe LA, et al. (2014) The recognition of and care seeking behaviour for childhood illness in developing countries: a systematic review. PloS one 9: e93427. [Crossref]

22. Wharton-Smith, A, Counihan, H and Strachan, C (2014) Implementing integrated community case management. Stakeholder experiences and lessons learned in three African countries. Malaria Consortium.

23. Republic of zambia ministry of health (2010) National Community Health Worker Strategy in Zambia. Republic of Zambia Ministry of Health.
24. Littrell M, Miller JM, Ndhlovu M, Hamainza B, Hawela M, et al. (2013) Documenting malaria case management coverage in Zambia: a systems effectiveness approach Malaria journal 12: 371

25. Littrell M, Gatakaa H, Evance I, Poyer S, Njogu J, et al. (2011) Monitoring fever treatment behaviour and equitable access to effective medicines in the context of initiatives to improve ACT access: baseline results and implications for programming in six African countries. Malar J 10: 327. [Crossref]

26. Kangwana BP, Kedenge SV, Noor AM, Alegana VA, Nyandigisi AJ, et al. (2011) The impact of retail-sector delivery of artemether-lumefantrine on malaria treatmen of children under five in Kenya: a cluster randomized controlled trial. PLoS Med 8: e1000437. [Crossref]

27. Burton DC, Flannery B, Onyango B, Larson C, Alaii J, et al. (2011) Healthcareseeking behaviour for common infectious disease-related illnesses in rural Kenya: a community-based house-to-house survey. J Health Popul Nutr 29: 61-70. [Crossref]

28. De Savigny D, Mayombana C, Mwageni E, Masanja H, Minhaj A, et al. (2004) Careseeking patterns for fatal malaria in Tanzania. Malar J 3: 27. [Crossref]

29. Rutebemberwa E, Kallander K, Tomson G, Peterson S, Pariyo G (2009) Determinants of delay in care-seeking for febrile children in eastern Uganda. Trop Med Int Health 14: 472-479. [Crossref]

30. Deressa W, Ali A, Berhane Y (2007) Household and socioeconomic factors associated with childhood febrile illnesses and treatment seeking behaviour in an area of epidemic malaria in rural Ethiopia. Trans R Soc Trop Med Hyg 101: 939-947. [Crossref]

31. Colvin C J, Smith HJ, Swartz A, Ahs JW, De Heer J, et al. (2013) Understanding care seeking for child illness in sub-Saharan Africa: a systematic review and conceptual framework based on qualitative research of household recognition and response to child diarrhoea, pneumonia and malaria. Soc Sci Med 86: 66-78. [Crossref]

32. Sasaki S, Fujino Y, Igarashi K, Tanabe N, Muleya CM, et al. (2010) Access to a health facility and care-seeking for danger signs in children: before and after a communitybased intervention in Lusaka, Zambia. Trop Med Int Health 15: 312-320. [Crossref]

33. GaoW, Dang S, Yan H, Wang D (2012) Care-seeking pattern for diarrhoea among children under 36 months old in rural western China. PloS one 7: e43103. [Crossref]

34. Page AL, Hustache S, Luquero FJ, Djibo A, Manzo ML (2011) Health care seeking behaviour for diarrhoea in children under 5 in rural Niger: results of a cross-sectional survey. BMC public health 11: 389. [Crossref]

35. Taffa N, Chepngeno G (2005) Determinants of health care seeking for childhood illnesses in Nairobi slums. Trop Med Int Health 10: 240-245. [Crossref]

36. Bryce J, Gilroy K, Jones G, Hazel E, Black RE, et al. (2010) The Accelerated Child Survival and Development programme in west Africa: a retrospective evaluation. The Lancet 375: 572-582.

37. Arifeen SE, Hoque DE, Akter T, Rahman M, Hoque M E, et al. (2009) Effect of the Integrated Management of Childhood Illness strategy on childhood mortality and nutrition in a rural area in Bangladesh: a cluster randomised trial. Lancet 374: 393-403. [Crossref]

38. Edward A, Dam K, Chege J, Ghee AE, Zare H, et al. (2016) Measuring paediatric quality of care in rural clinics-a multi-country assessment-Cambodia, Guatemala, Zambia and Kenya. Int J Qual Health Care 28: 586-593. [Crossref]

39. Hamooya BM, Chongwe G, Dambe R, Halwiindi H (2016) Treatment-seeking behaviour for childhood fever among caretakers of Chivuna and Magoye rural communities of Mazabuka District, Zambia: a longitudinal study. BMC public health 16: 762. [Crossref]

Copyright: (C2018 Edward A. This is an open-access article distributed under the terms of the Creative Commons Attribution License, which permits unrestricted use, distribution, and reproduction in any medium, provided the original author and source are credited. 